\title{
STATISTICAL ANALYSIS OF THE FORENSIC AUTOPSIES, PERFORMED IN THE DEPARTMENT OF FORENSIC MEDICINE OF UNIVERSITY HOSPITAL - PLEVEN FOR THE PERIOD 2009-2013 (A PRELIMINARY REPORT)
}

\author{
Dancho P. Dekov, \\ Ivan N. Ivanov', \\ Sergey D. Kostadinov, \\ Savelina L. Popovska ${ }^{1}$, \\ Petko I. Lisaev, \\ Plamen D. Dorovski
}

Department of General Medicine, Forensic Medicine and Deontology, Faculty of Public Health, Medical University - Pleven

${ }^{1}$ Department of Pathology, Faculty of Medicine, Medical University - Pleven

\section{Corresponding author:}

Dancho P. Dekov

Medical University - Pleven

1, St. Kliment Ohridski Str.

Pleven, 5800

e-mail:drdekov@abv.bg

Received: November 02, 2014

Revision received: March 07, 2016

Accepted: May 30, 2016

\section{Summary}

The study is a statistical analysis of the dynamics and structure of forensic autopsies performed on dead bodies and body remains at University Hospital "Dr. Georgi Stranski" in Pleven. The study was based on forensic autopsy records database and forensic autopsy reports for the period 2009-2013. A total of 976 cases, including forensic autopsies, exhumations, examination of bone remains and inspection of dead bodies at the site of death (without following forensic autopsy), were analyzed. The studied data was coded and processed with Microsoft Excel software. A detailed data analysis of the number of autopsies, frequency of death by cause, distribution of death by gender, age, residence, place of death and its forensic category is presented. According to our results, the deaths due to diseases comprised $35 \%$ of all forensic autopsies. The most common cause of violent death was damage by mechanical factors $(53 \%)$, followed by asphyxia $(24.7 \%)$. The most common mechanical factor was vehicle crash trauma, followed by falling $(22.5 \%)$. Accidents were the most common cause of death $62 \%$, followed by suicides (31\%) and homicides 7\%. Fifty-nine percent of the suicides were by hanging. The preliminary analyses of the studied data lead to several conclusions. The number of forensic autopsies was found to be progressively decreasing, but the road traffic death rate remained unchanged. Most of the forensic autopsies were performed in July. The highest death rates by age was observed in the age group between 51 and 60 years, with predominance of males over females (3.3 males: 1 females).

Key words: forensic medicine, mortality determinants, analysis

\section{Introduction}

During the last two decades, the number of pathological autopsies has been continuously decreasing. There are many reasons for the decrease, including availability of contemporary, sophisticated diagnostic imaging technologies, refusal of autopsy due to cultural or religious reasons, or certain economic factors. The number of forensic autopsies depends on the legislation of the particular country. Interestingly, autopsy was 
performed of only one out of every ten dead people in 2007 in the USA, nine out of ten cases being violent deaths [1].

The aim of the investigation was to study the number, dynamics, causes and characteristics of deaths, based on forensic autopsies over a fiveyear period in the Pleven region.

\section{Materials and Methods}

The study was based on forensic autopsy records database and forensic autopsy reports for the period 2009-2013. A total of 976 cases, including forensic autopsies, exhumations, examination of bone remains and inspections of dead bodies at the site of death (without following forensic autopsy) were analyzed. The data studied was coded and processed with Microsoft Excel software. A detailed data analysis of the number of autopsies, frequency of death by cause, distribution of death by gender, age, residence, place of death and its forensic category is presented.

\section{Results}

The studied cases included 976 forensic autopsies of dead bodies and body parts. In 903 cases, a detailed inspection of the body and forensic autopsy were performed. In 58 cases only inspection of the dead body was performed in the presence of an investigating officer. In these cases, death certificates were issued by a family doctor. Two exhumations and 13 cases of examination of bone remainings were performed during the studied period.

The distribution of forensic autopsies during the five-year period is presented on Figure 1. There was not a clear tendency towards decrease in the rates of forensic autopsies, despite the fact that the lowest rates were documented in 2013. Forensic autopsies were performed far more often on male than on female subjects (Figure 2).

The highest mortality (autopsy) rate, according to 961 forensic autopsies, was observed in the age group between 41 and 80 years (Figure 3 ).

In 599 cases (62. 8\%), forensic autopsies were performed on city dwellers. Three-hundred and fifty-five of the forensic autopsies were performed on people from rural areas. No data was available for some of the cases.

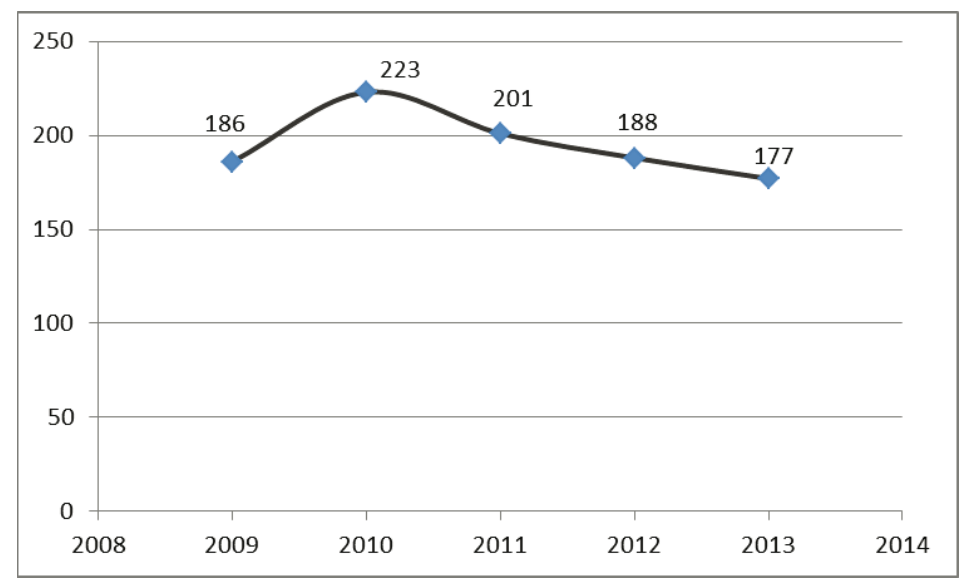

Figure 1. Rates of autopsies per year (for the studied period)

According to forensic autopsies, the most common cause of death was trauma (violent death), followed by sudden death (unexpected death, caused by a disease). The causes of death are presented in details on Figure 4. The analyses of the deaths caused by mechanical trauma showed a significant predominance of vehicular (car) traumas and falls from height and from a person's own height. Details, concerning the number and distribution of mechanical traumas by type are presented on Figure 5.

According to data from forensic autopsies, there was no significant change in the number of deaths per year, caused by vehicular accidents during the studied period (Figure 6).

According to forensic autopsies data, most of the cases of violent death were caused by accidents. Details are presented on Figure 7. 


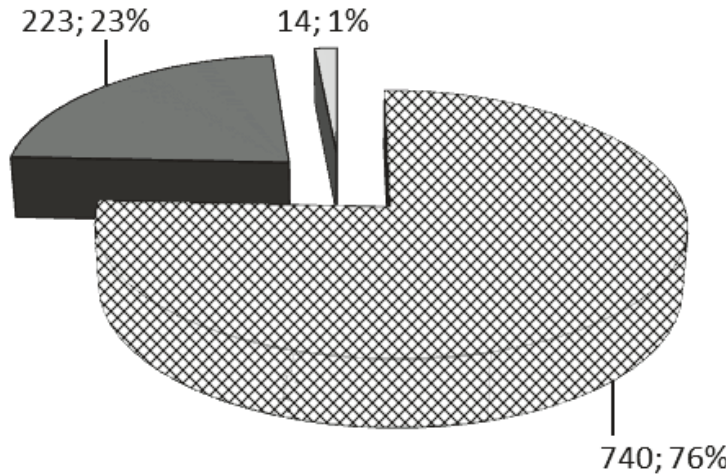

囚Male

口Female

No data

Figure 2. Distribution of autopsy cases by gender

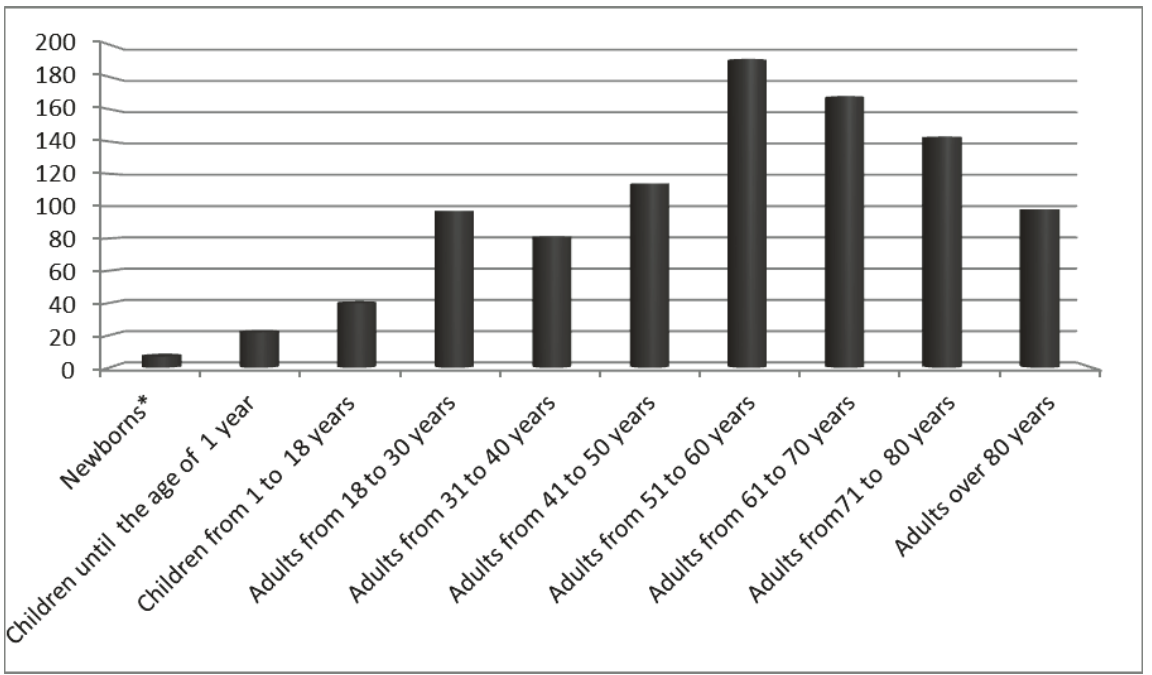

*Each child during the first 24 hours of life was considered as newborn

Figure 3. Death rates by age

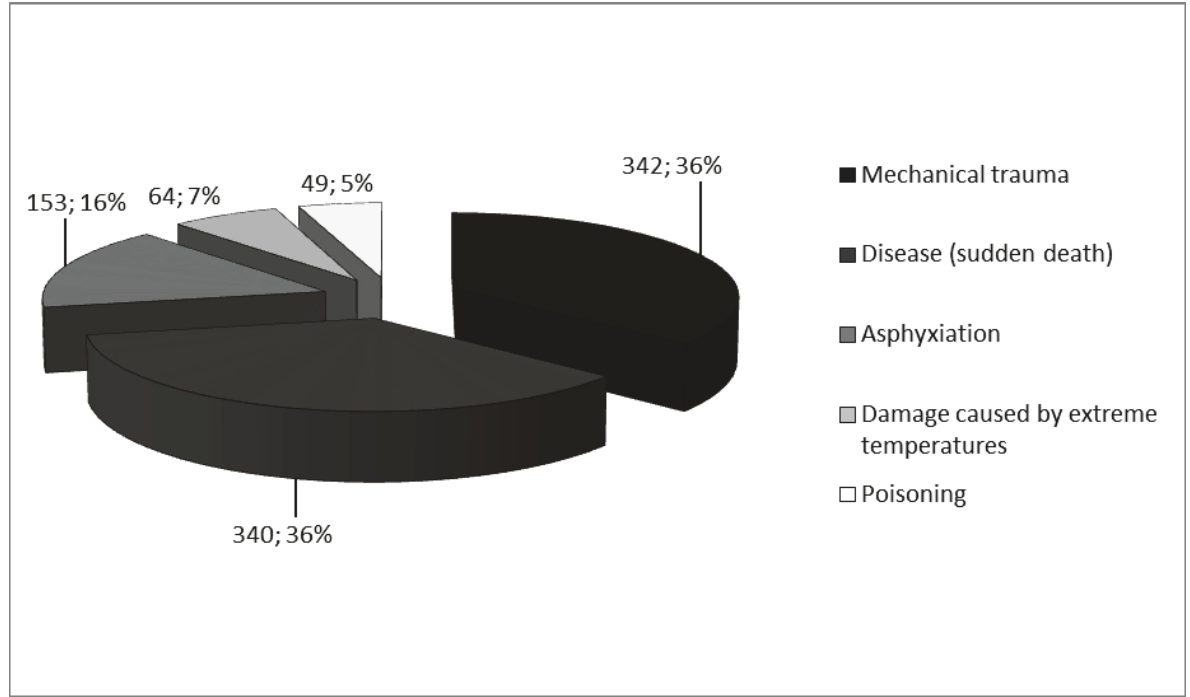

Figure 4. Causes of death, according to results from forensic autopsies 


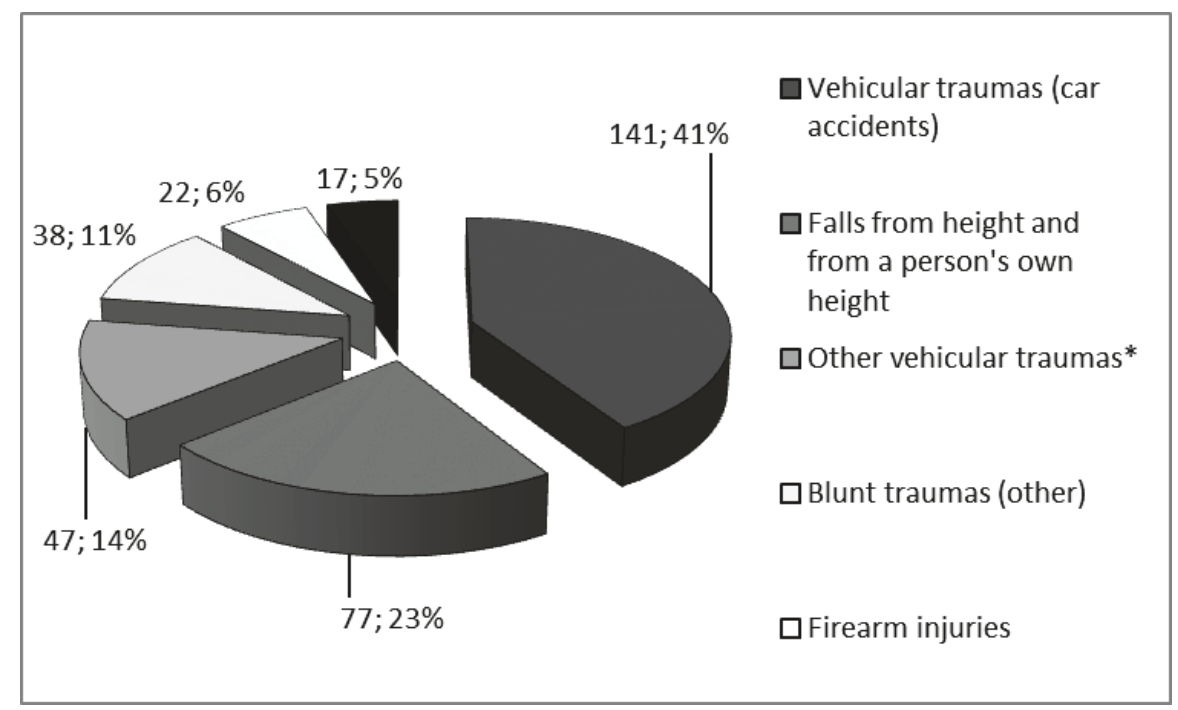

*Other vehicular traumas - railway, motorcycle, bicycle and caterpillar/wheeled tractor accidents

Figure 5. Structure and incidence of mechanical traumas

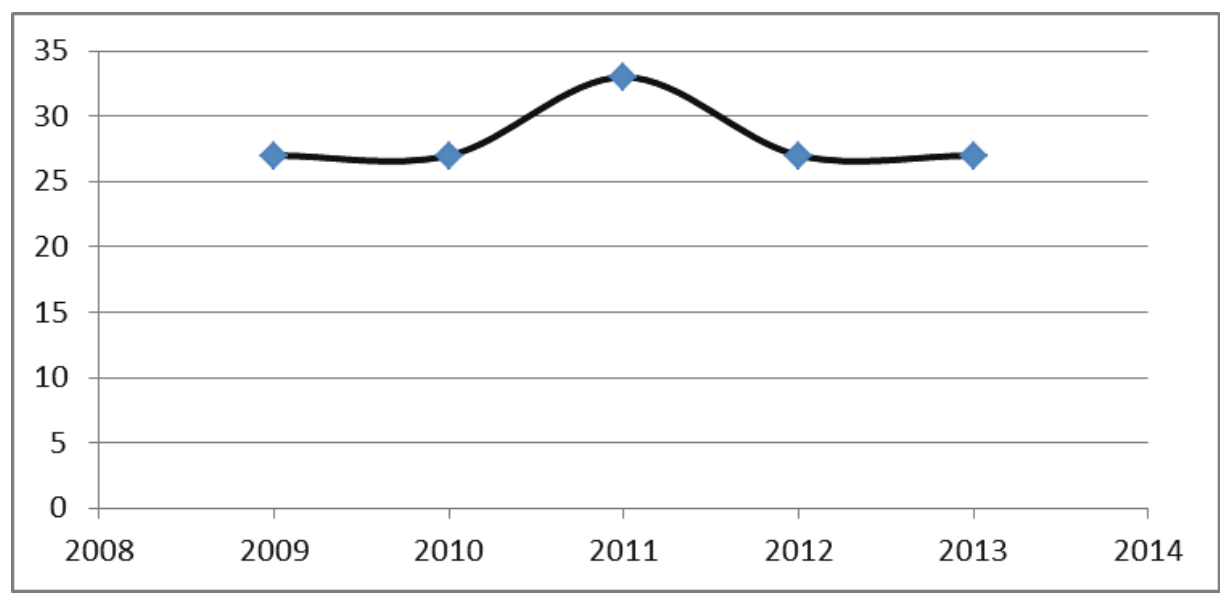

Figure 6. Deaths caused by vehicular traumas (car accidents in particular) per year and dynamics of incidence for the studied period

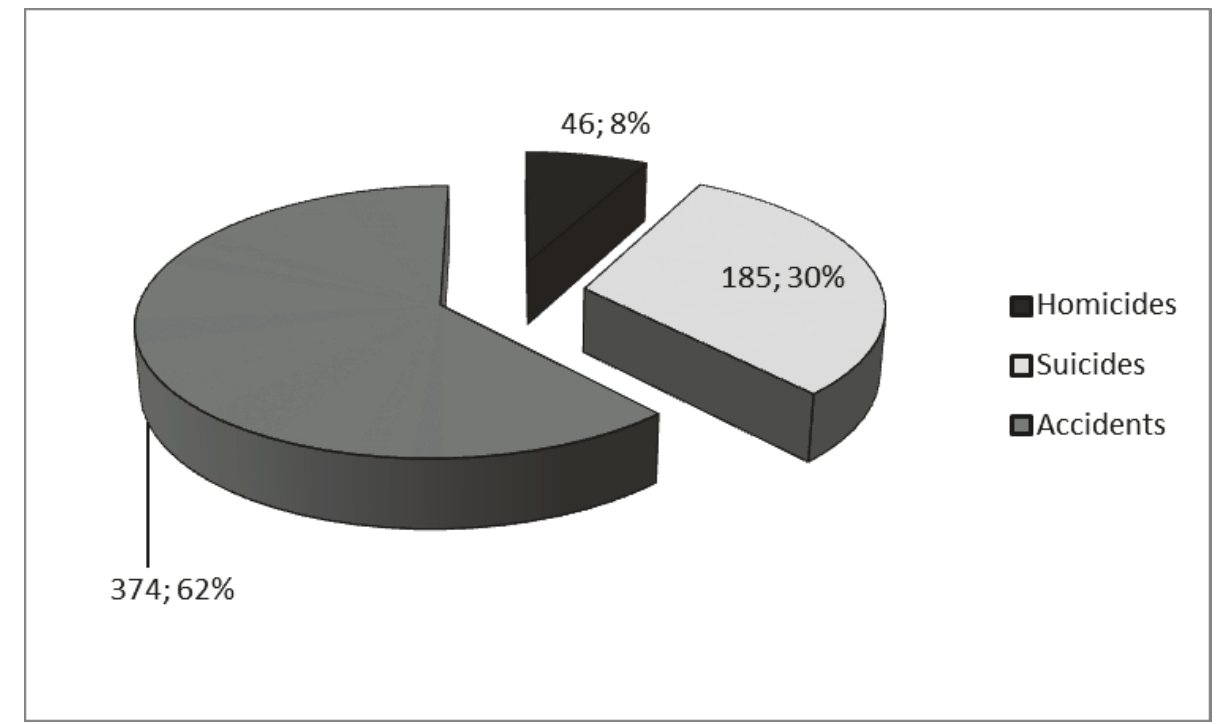

Figure7. Types of the violent death 
The overall number of deaths, due to violent causes was 605 . The number of sudden deaths (unexpected death, caused by a disease) was 340 . In 31 of the studied cases, the cause of death remained unidentified.

The number of forensic autopsies per month (calculated for the studied period), demonstrated some variations. The highest number of autopsies was documented for June, July, January and March. Details are presented on Figure 8.

In 150 cases $(16.6 \%)$, alcohol in concentrations higher than $0.5 \%$ were found in the urine and blood samples collected from the bodies. This was considered as a sign of alcohol consumption before the fatal outcome of a subject. In 43 cases the concentration of alcohol in the blood was 0.5 to $1.5 \%$, in 45 cases the concentration was between 1.5 and $2.5 \%$, and in 50 cases it was between 2.5 and $4.0 \%$. Twelve of the cases were found to be with severe (potentially lethal) alcohol intoxication concentration of alcohol in the blood more than $4 \%$.

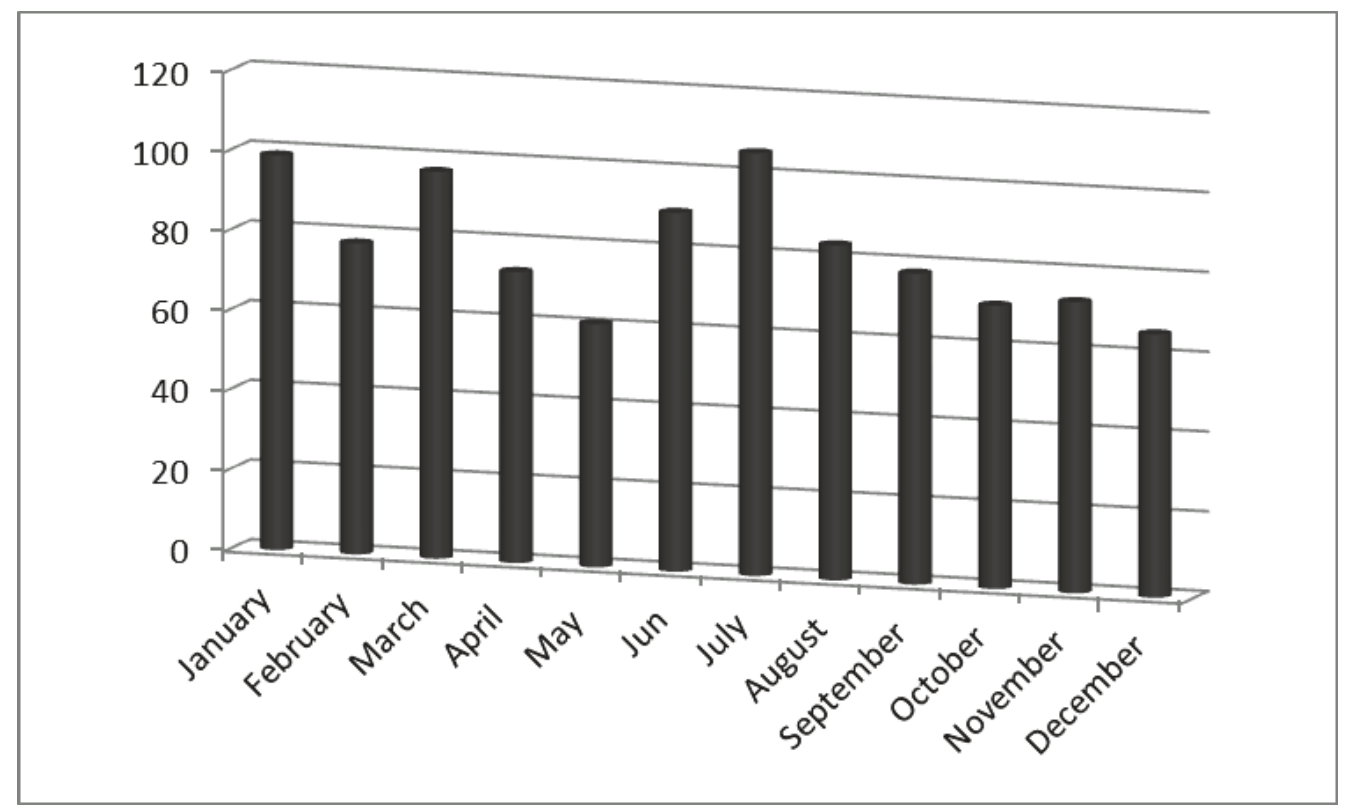

Figure 8. Number of forensic autopsies per month (calculated for the studied period)

\section{Discussion}

The most important task of a forensic autopsy is to identify the cause of death and confirm or exclude violent death. This information is particularly important for investigating each suspicious case.

The number of forensic autopsies per year was found to show some increase (with approximately $20 \%$ ) during the period 20092010 , followed by a gradual decrease during the following three years. This tendency towards a slight decrease in the autopsy rates per year was also observed in pathological autopsies for the same area during approximately the same period [2].

Of particular interest is the fact, that the number of autopsies in the Republic of Turkey shows significant decrease during the months of
Ramadan [3]. According to our data, most autopsies per month were performed in July - 106 cases (10. 9\%), followed by January - 99 cases $(10.1 \%)$. The months with the smallest number of autopsies were May (61 - 6. 3\%) and December (66-6. 8\%). We think that we cannot compare our results with the ones cited above, because of the relative religious heterogeneity of our population and the lack of clear information concerning the actual part of population who keep the fast.

Our results demonstrate that the fatalities due to non-violent causes (caused by disease) represented about $35 \%$ of the deaths confirmed by forensic autopsies.

Among the most common causes of violent death were mechanical traumas (53\%), followed by asphyxiation $(24.7 \%)$. According to forensic 
autopsies, the leading cause of mechanical traumas were vehicular accidents $(42.3 \%)$, followed by falls from height $(22.5 \%)$. According to Bulgarian legislation, every victim of a vehicular accident undergoes forensic autopsy $[4,5]$.

In Norway, forensic autopsy might be avoided in some of the cases of vehicular trauma fatalities. According to Igeltjørn M. and Nordrum I.S., forensic autopsies were performed on $63 \%$ of the victims of road accidents in the region of Norway for the period 1996-2005. The authors observed some differences in the frequency of forensic autopsies in different areas included in their study. They attributed these differences to differences in understanding the importance of forensic autopsies, economic backgrounds and differences in interpretation of law and lawsuit process [6].

It is also interesting to note, that the vehicular accident fatality rate in Norway in 2007 was 48.8 per 1000000 citizens, whereas it was 131.7 per 1 000000 in Bulgaria for the same year [7]. The large number of forensic autopsies per year in Bulgaria is related to the number of vehicular accident fatalities and current Bulgarian legislation. Accidents (including vehicle, occupational and domestic accidents) were found to be the leading cause of violent death, representing $62 \%$ of all cases of violent death for the period studied. Suicides were the second most common cause of violent death observed in $31 \%$ of the cases, while homicides represented accounted for only $7 \%$ of all forensic autopsies during this period.

Fifty-nine percent of the committed suicides were performed by hanging. Despite the fact that there are some differences in the suicide methods used in different countries, the most common method is usually suicide by hanging [8]. Our data also proved that suicides were most commonly committed by hanging.

According to our results, forensic autopsies were performed most often on individuals in the age group 51-60, followed by those aged 61-70. According to data for approximately the same period, pathological autopsies were performed most often on individuals in the age group 71-80 years, followed by those in the age group 61-70 years [2]. This difference is attributable to the fact that pathological autopsies were performed on subjects, who died from natural causes at a relatively older age, as compared to the individuals who died from violent death, often at a younger age.
The ratio of forensic autopsies performed on male subjects to that performed on female subjects was 3.3:1. The ratio of male to female subjects that were subjected to pathological autopsy for approximately the same period was 1.6:1 [2]. These results can partly be explained by the differences in causes of death elicited by forensic and pathological autopsies. Canturk N. et al. reported the same tendency towards predominance of male subjects in a series of forensic autopsies. These authors also observed significant variations in the male-female ratio among different age groups [3].

\section{Conclusions}

The preliminary analysis of the studied forensic autopsy cases led to conclusions that the number of forensic autopsies per year gradually decreased, while the number of deaths per year caused by vehicular traumas remained almost the same during the five-year period, and the highest total autopsy rate per month was observed in July. Our results demonstrated that forensic autopsies were performed most often on individuals in the age group 51-60. The ratio of forensic autopsies performed on male subjects to that performed on female subjects was 3.3:1.

\section{References}

1. Hoyert DL. The changing profile of autopsied deaths in the United States, 1972-2007. NCHS Data Brief [Internet]. 2011 [cited 2014 Oct 31];67:1-8. Available from: http://www.cdc.gov/nchs/data/databriefs/db67.pdf

2. Ivanov I, Dekov D, Popovska S. [Statistical analyses of the results from pathologoanatomic autopsy performed in the Department of Pathology (University Hospital Pleven) during the period 2008-2012. (A preliminary report)]. Publications of the Bulgarian Union of Scientists - Varna. 2013;18:79-84. Bulgarian.

3. Canturk N, Turkmen N, Canturk G, Dagalp R. Differences in the number of autopsies and causes of death between the months of Ramadan and control months and between two cities, Ankara and Bursa in Turkey. Med Glas (Zenica). 2013;10(2):354-8.

4. Penal Procedure Code of Republic of Bulgaria. Sofia: Sibi; 2014.

5. Ordinance No. 2 of Oct 26, 2011: Published by Ministry of Justice, Ministry of Interior and Ministry of Health. State Gazette. 2011 Nov 18:91. 
6. Igeltjørn M, Nordrum IS. Frequency of forensic autopsies after deaths in road traffic accidents. Tidsskr Nor Laegeforen. 2009;12(18):1850-2. doi: 10.4045/tidsskr.08.0274.

7. State-Public Consultative Commission on the Problems of Road Safety [Internet]. Sofia:
Ministry of Interior [cited 2015 Oct 31]. Road accident statistics. Available from: http://dokkpbdp.mvr.bg/Statistics/default.htm

8. Reddy MS. Suicide incidence and epidemiology. Indian J Psychol Med. 2010;32(2):77-82. doi: 10.4103/0253-7176.78501. 